\title{
Systematic study on charge transport and spectroscopic properties: A DFT Benchmark of LCD's molecules
}

\author{
Felipe B. C. Marinho* (IC), Sidney R. Santana (PQ); \\ Depart. of Chemistry and Physics, Center of Agrarian Center, Federal University of Paraíba (UFPB). \\ e-mail: santanasidney@cca.ufpb.br
}

Keywords: DFT, LCD, Benchmark.

\section{Introduction}

Nowadays we have been faced with a large quantity of papers, works and device due the technological improvements of TV's and monitors, OLED's and LCD's, in the last years[1]. So, in this work we have been investigated LCD's molecules focusing the development of a database of electron/hole transfer and spectroscopic properties[2,3]. In this sense, a DFT benchmark has been created to evaluate these properties that have a large dependence of functionals and basis sets.

\section{Methodology}

We have been chosen M06-2X, w-B97XD, CAMB3LYP and B3LYP functionals and $6-31+\mathrm{G}^{*}, 6-31+\mathrm{G}^{* *}$ basis sets to describe molecules based in branched mesoionic centers to generate a discotic-like molecular electronic devices[4] considering $\mathrm{Alq}_{3}$ such as the reference molecule[2,3].

\section{Results}

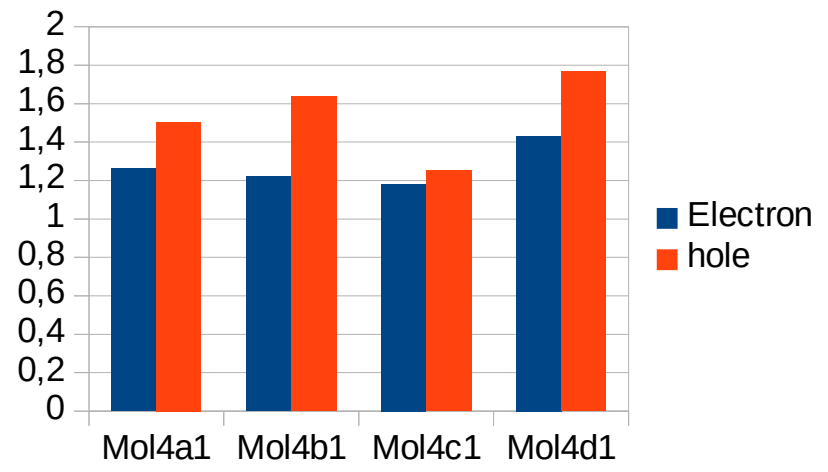

Figure 1: Results of Lambda (electron or hole) to B3LYP/6-31+G*.

\section{Discussion}

In figure 1, all the molecules shown reasonable results to electron transfer properties when compared with $\mathrm{Alq}_{3}$ reference, with lambda hole and electron one greater than 1.2 times than that.

\section{Conclusions}

Through these data, we could modeling molecules that could employed in this study to be great candidates to best electron/hole transfers new materials. 


\section{Acknowledgments}

CAPES, CNPq, UFPB, Cenapad/SP

\section{References}

[1] http://www.trustedreviews.com/opinions/oled-vs-led-lcd [2] J. L. Rao; et. al.; Theor. Chem. Acc. 129 (2011) 131-139

[3] J. C. S. Costa, et. al., J. Phys. Chem. C 118 (2014) 21762.

[4] T. Yasuda; J. Am. Chem. Soc. 2011, 133, 13437-13444 